\title{
The RET polymorphic allele S836S is associated with early metastatic disease in patients with hereditary or sporadic medullary thyroid carcinoma
}

\author{
Débora $R$ Siqueira, Mírian Romitti, Andreia P da Rocha, Lucieli Ceolin, \\ Camila Meotti, Aline Estivalet, Marcia K Puñales and Ana Luiza Maia
}

\author{
Endocrine Division, Thyroid Section, Serviço de Endocrinologia, Hospital de Clínicas de Porto Alegre, Universidade Federal do Rio \\ Grande do Sul, Rua Ramiro Barcelos 2350, 90035-003 Porto Alegre, RS, Brazil \\ (Correspondence should be addressed to A L Maia; Email: almaia@ufrgs.br)
}

\begin{abstract}
The possible role of $R E T$ variants in modifying the natural course of medullary thyroid carcinoma (MTC) is still a matter of debate. Here, we investigate whether the RET variants L769L, S836S, and G691S/S904S influence disease presentation in hereditary or sporadic MTC patients. One hundred and two patients with hereditary MTC and 81 patients with sporadic MTC attending our institution were evaluated. The frequencies of $R E T$ polymorphisms in hereditary MTC were as follows: L769L, 17.3\%; S836S, 7.95\%; and S904S/G691S, 18.2\%. No associations were observed between these polymorphisms and pheochromocytoma, hyperparathyroidism, lymph node, or distant metastasis. However, patients harboring the S836S variant were younger than those without this allele $(17 \pm 8.2$ vs $28.6 \pm 14.4$ years, $P=0.01)$, suggesting that these patients had metastases at a young age. Accordingly, the cumulative frequency of local and/or distant metastases as estimated by Kaplan-Meier curves showed that lymph node and distant metastases occurred earlier in patients harboring the S836S variant $(P=0.003$ and $P=0.026$ respectively). The S836S allele frequency was higher in sporadic MTC patients than in controls (10.5 vs $3.1 \%$, $P=0.01)$. Individuals harboring the S836S variant were younger ( $38.6 \pm 13.3$ vs $48.5 \pm 16.7$ years, $P=0.02)$ and showed a higher percentage of lymph node and distant metastases $(P=0.02$ and $P=0.04$ respectively). Kaplan-Meier estimates of lymph node and distant metastases yielded distinct curves for patients with or without the S836S allele $(P=0.002$ and $P=0.001$ respectively). Additional analyses using a COX regression model showed that the S836S variant was independently associated with metastatic disease (hazard ratio 2.82 (95\% confidence interval $1.51-5.26), P=0.001)$. In conclusion, the RET S836S variant is associated with early onset and increased risk for metastatic disease in patients with hereditary or sporadic MTC.
\end{abstract}

Endocrine-Related Cancer (2010) 17 953-963

\section{Introduction}

Medullary thyroid carcinoma (MTC), a malignant neoplasia of the parafollicular $\mathrm{C}$ cells of the thyroid, may occur sporadically or as part of the inherited cancer syndrome multiple endocrine neoplasia type 2 (MEN 2; Kouvaraki et al. 2005). The MEN 2 syndrome includes three clinically distinct forms: MEN 2A, MEN 2B, and familial MTC (FMTC). In patients with FMTC, only the thyroid is affected. Patients with MEN 2A develop MTC, pheochromocytoma (PHEO), and/or primary hyperparathyroidism (HPT). MEN 2B patients have MTC, PHEO, ganglioneuromas of the digestive tract, mucosal neuromas, and/or skeletal abnormalities.

The RET proto-oncogene is the susceptibility gene for hereditary MTC, and recent studies showed a timedependent MTC progression, strengthening the importance of a DNA-based RET genotype analysis for the identification of asymptomatic gene carriers at risk of developing MTC (Machens et al. 2003, Punales et al. 2008). Gain-of-function germline mutations in MEN 2A 
and FMTC syndromes have been described in RET exons 5, 8, 10, 11, 13, 14, and 15 (Kouvaraki et al. 2005). However, the majority of MEN 2A families have mutations of one of the five conserved cysteine residues in exon 10 (codons 609, 611, 618, and 620) or exon 11 (codon 634) in the extracellular domain of RET (Eng et al. 1996, Ponder 1999). The presence of any mutation at codon 634 has been associated with the presence of PHEO and HPT. Conversely, mutations at codons 768 and 804 are thus far associated with FMTC. The reasons for these genotype-phenotype correlations have not been completely clarified yet. Although the different levels of RET activation induced by the different mutations could partially explain the phenomena, the observed clinical variability and aggressiveness in members of the same family suggest a role for genetic modifiers in the clinical course of MTC (Ponder 1999, Machens et al. 2001, Robledo et al. 2003).

The possible role of neutral RET sequence variants in modifying the MEN 2 clinical course or MEN 2-related tumors is still a matter of debate. Some studies have shown that RET single nucleotide polymorphisms (SNPs) could interfere in the disease presentation of hereditary MTC syndromes (Magalhaes et al. 2004, Rocha et al. 2007). Robledo et al. (2003) demonstrated that two of these RET variants (G691S and S904S) may modify the age at onset of MTC tumor in family members, although these findings could not be replicated in a large European population sample (Lesueur et al. 2006). It was also suggested that the L769L polymorphism might contribute to the earlier onset of MTC in a patient with a V804M mutation (Magalhaes et al. 2004). Recently, an association was described between two RET variants, IVS1-126G $>\mathrm{T}$ and IVS8 $+82 \mathrm{~A}>\mathrm{G}$; 85_86insC, with the clinical course of hereditary MTC in a six-generation family with a G533C RET mutation (Tamanaha et al. 2009). Nevertheless, other studies failed to demonstrate any effect of RET polymorphisms on the natural course of hereditary MTC (Fernandez et al. 2006a, Lesueur et al. 2006).

The purpose of this study was to investigate whether the RET neutral variants G691S, L769L, S836S, or S904S influence the clinical presentation and disease outcome in a large cohort of individuals with MEN 2A. These polymorphisms were selected based on their previous association with the clinical course of hereditary or sporadic MTC (Robledo et al. 2003, Wiench et al. 2004, Baumgartner-Parzer et al. 2005, Lesueur et al. 2006). We observed that the S836S polymorphism was associated with younger age at diagnosis and early metastatic disease in hereditary disease. Therefore, we have decided to evaluate whether these findings would be evident in sporadic MTC.

\section{Materials and methods}

\section{Patients}

Patients with a diagnosis of hereditary MTC attending the Endocrine Division at Hospital de Clínicas de Porto Alegre were invited to participate in the study. Since 1997, our division has been a reference center for the molecular testing of RET germline mutations in Brazil, and therefore patients referred to us by other Brazilian centers for molecular investigation were also invited to participate. All patients and/or their legal guardians provided written consent in accordance with the institutional Ethics Committee.

The data collected for each individual included the clinical characteristics of family members (association of other endocrine neoplasias), the type of RET mutations, and information on atypical features noted, such as Hirschsprung's disease (HIRS) or cutaneous lichen amyloidosis (CLA). Patients underwent a complete clinical examination, laboratory tests (levels of basal calcitonin (Until December 2003, Calcitonin IRMA-DSL7700, Diagnostic Systems Laboratories, Inc., Webster, TX, USA, reference range $<10 \mathrm{pg} / \mathrm{ml}$ and, after January 2004, Immulite 2000, Diagnostic Products Corporation, Los Angeles, CA, USA; reference value (VR) male $<12.0 \mathrm{pg} / \mathrm{ml}$ and female $<6.0 \mathrm{pg} / \mathrm{ml}$ )), plasma parathyroid hormone (PTH; Immulite 2000 Intact PTH, Diagnostic Products), urinary fractionated metanephrines (HPLC), and, whenever indicated, diagnostic imaging investigation (cervical ultrasonography, thorax and abdominal computed tomography (CT)). Selected patients were submitted to whole-body metaiodobenzylguanidine scintigraphy to rule out PHEO and/or distant metastasis.

Our initial sample comprised 102 patients, with germline mutations of the RET proto-oncogene and/or immunohistochemistry diagnosis of MTC, who were identified by genetic screening at our institution, belonging to 17 unrelated families with MEN 2A and its variants or FMTC. Of them, 68 patients were diagnosed based on clinical evidence of disease, and 34 gene carriers were identified through genetic screening. Subjects who presented with physical signs compatible with MTC (palpable thyroid nodule and/or lymph node enlargement) at diagnosis were considered as presenting clinical disease, and individuals without physical disease were considered as asymptomatic gene carriers. Fourteen of these patients were excluded, either because they were awaiting surgery ( 2 patients) or not enough material was available for polymorphism analysis (11 patients). We also excluded one patient with a mutation at RET codon 768 , exon 13 , 
$\mathrm{Glu} \rightarrow$ Asp (E768D) due to the characteristic low-risk disease phenotype.

We also evaluated 81 patients with sporadic MTC. The diagnosis of sporadic MTC was based on the histopathological/immunohistochemistry findings and the absence of known germline RET point mutations in exons $8,10,11$, or $13-16$. The clinical and laboratorial data were collected for each individual in the sporadic group. A group of 80 health volunteers attending the blood donation facility of Hospital de Clínicas de Porto Alegre (Porto Alegre, Brazil) constituted our control group. A standard questionnaire was used to collect information about age, sex, skin color, and history of neoplasias.

Total thyroidectomy was performed in all patients with varying cervical neck dissection procedures. The diagnosis of lymph node metastasis was based on histological examination. Patients with suspicious distant metastasis (i.e. the presence of local metastases and/or serum calcitonin $>150 \mathrm{pg} / \mathrm{ml}$ ) underwent imaging exams (cervical, thoracic and abdomen CT (or liver magnetic resonance imaging), and bone scintigraphy). Patients with undetectable calcitonin levels were considered free of disease.

Patients with PHEO or HPT underwent specific surgery. Tumor staging was performed according to the current International Union against Cancer TNM classification (O'Sullivan \& Shah 2003).

\section{Single nucleotide polymorphism analysis}

The following RET SNPs were selected based on their previous association with the clinical course of sporadic or hereditary MTC: G691S (codon 691 of exon 11, GlyGGT $\rightarrow$ SerAGT), L769L (codon 769 of exon 13, LeuCTT $\rightarrow$ LeuCTG), S836S (codon 836 of exon 14, SerAGC $\rightarrow$ SerAGT), and S904S (codon 904 of exon 15, SerTCC $\rightarrow$ SerTCG). For genotyping, genomic DNA was prepared from peripheral blood leukocytes by standard procedures, and the fragments covering the RET variants were amplified using the PCR primers and conditions previously described (Punales et al. 2003). Genotyping was performed using either restriction fragment length polymorphism (RFLP) or direct sequencing. For RFLP analysis, an aliquot of PCR product was digested with the appropriate restriction enzyme and analyzed as previously described (Punales et al. 2003). For sequencing, PCR products were purified using the GFX PCR DNA purification kit (GE Healthcare, Buckinghamshire, UK) and submitted to direct sequencing using the Big Dye Terminator Cycle Sequencing Ready Reaction kit (Applied Biosystems, Foster City, CA, USA).

\section{Somatic M918T mutation analysis}

For sporadic patients, we analyzed the frequency of somatic M918T mutations. The MTC samples were material paraffin-embedded formalin-fixed tissue blocks. DNA was extracted using the Magnesil Genomic Fixed Tissue System (Promega, Madison, WI, USA) according to the manufacturer's instructions. Exon 16 was amplified by PCR using $100-300 \mathrm{ng} / \mu \mathrm{l}$ of DNA in a reaction mix $(25 \mu \mathrm{l})$ containing $20 \mathrm{mM}$ Tris$\mathrm{HCl}, \mathrm{pH} 8.0,50 \mathrm{mM} \mathrm{KCl}, 2 \mathrm{mM} \mathrm{MgCl}_{2}, 0.2 \mathrm{mM}$ dNTPs, $0.2 \mathrm{mM}$ of each primer, and $1.25 \mathrm{U}$ Platinum Taq DNA Polymerase (Invitrogen Life Technology, Carlsbad, CA, USA). The running profile of the amplification and RFLP analysis were similar to those described for genomic DNA (Punales et al. 2003).

\section{Statistical analysis}

Results are expressed as mean \pm s.D. or median and interquartile intervals unless otherwise specified. Baseline characteristics were compared using the $\chi^{2}$-test or Fisher's exact test for qualitative variables, or the Student's $t$-test or Mann-Whitney's $U$ test for quantitative variables. Hardy-Weinberg equilibrium for each polymorphism was assessed by the Fisher's exact test. The differences in cumulative lymph node and/or distant metastasis between groups were tested by Kaplan-Meier curves; comparisons between curves were performed using the log rank test. We performed a Cox regression model to investigate the effect of several variables upon the time of a specified event: the presence of metastasis. The Statistical Package for the Social Sciences 15.0 (SPSS Inc., Chicago, IL, USA) was used, and $P<0.05$ was considered as statistically significant.

\section{Results}

\section{Frequency of RET polymorphisms in MEN 2A patients}

Table 1 shows the clinical and molecular data of the families with MEN 2A. Of the 17 independent families with hereditary MTC analyzed, 13 were classified as MEN 2A, 3 classified as MEN 2A associated with CLA, 1 associated with HIRS, and 1 classified as FMTC. All but four MEN 2A/FMTC kindred had a mutation at RET codon 634 in exon 11, the most prevalent mutation accounting for $89 \%$ of cases. The identified mutations were as follows: Cys $\rightarrow \mathrm{Tyr}$ (C634Y, 65.9\%), Cys $\rightarrow \operatorname{Arg}$ (C634R, 15.4\%), and $\mathrm{Cys} \rightarrow \operatorname{Trp}(\mathrm{C} 634 \mathrm{~W} 7.7 \%)$. 
Table 1 Clinical presentation and RET germline mutations in multiple endocrine neoplasia 2A patients

\begin{tabular}{|c|c|c|c|c|c|c|c|}
\hline Phenotype & $N$ families & RET mutation & $\begin{array}{c}\text { Affected } \\
\text { individuals }\end{array}$ & $\mathrm{CCH}$ & MTC & PHEO & HPT \\
\hline \multirow[t]{4}{*}{ MEN $2 A$} & 6 & C634Y & 33 & 2 & 31 & 11 & 5 \\
\hline & 4 & C634R & 8 & & 8 & 5 & 3 \\
\hline & 1 & C634W & 7 & & 7 & 3 & 3 \\
\hline & 2 & C618R & 8 & & 8 & 2 & 0 \\
\hline \multirow[t]{2}{*}{ MEN 2A + CLA } & 2 & C634R & 6 & & 6 & 2 & 0 \\
\hline & 1 & C634Y & 25 & & 25 & 3 & 5 \\
\hline MEN 2A + HIRS & 1 & C618R & 1 & & 1 & 1 & 0 \\
\hline Total & 17 & & 88 & 2 & 86 & 27 & 16 \\
\hline
\end{tabular}

MTC, medullary thyroid carcinoma; PHEO, pheochromocytoma; HPT, hyperparathyroidism; CCH, C-cell hyperplasia; CLA, cutaneous lichen amyloidosis; HIRS, Hirschsprung's disease.

The allele frequencies of the RET polymorphisms are shown in Table 2. The observed SNP frequencies were similar to those reported in the literature (Elisei et al. 2004, Wiench et al. 2004, Baumgartner-Parzer et al. 2005, Wohllk et al. 2005, Severskaya et al. 2010). Confirming previous studies, the two variants G691S and S904S were in linkage disequilibrium, and, therefore, to avoid redundant information, the results were grouped together and referred as G691S/S904S (Robledo et al. 2003, Elisei et al. 2004, Tamanaha et al. 2009). All genotypes analyzed were in Hardy-Weinberg equilibrium $(P>0.20)$.

\section{Clinical and oncological features of hereditary MTC patients}

The clinical and oncological features of the subjects are listed in Table 3. The median basal serum calcitonin level at diagnosis was 140 (30-988.6) pg/ml. At first, we assessed whether the polymorphisms could have an effect on the age at onset of disease. Analysis of RET variants failed to demonstrate differences in the age of diagnosis related to the presence or the absence of the $L 769 L$ or G691S/S $904 \mathrm{~S}$ polymorphic allele. However, patients harboring the S836S variant were significantly younger than those without this allele $(17.0 \pm 8.2$ vs $28.6 \pm 14.4$ years, $P=0.01)$. There were no differences in basal serum calcitonin at diagnosis between individuals with or without S836S polymorphic allele (15 (8-344) vs 152 (36.9-1025) $\mathrm{pg} / \mathrm{ml}, P=0.25$ ).

It is reasonable to speculate that in those patients identified through molecular diagnosis, the natural course of the disease was interrupted by this intervention, and that the age of diagnosis would be lower than that observed in those individuals in whom the disease evolved naturally. This could be a confusing factor, particularly when analyzing age at onset of the disease. Therefore, we also analyzed both groups separately to avoid selection bias. Table 4 shows the effect of RET polymorphisms on MTC presentation in individuals diagnosed by genetic screening or clinical evidence of disease. There were no differences in age or serum calcitonin levels (37.5 (9.9-73) vs $9.5(7.1-24.1) \mathrm{pg} / \mathrm{ml}, P=0.11)$ at diagnosis between individuals with or without polymorphic alleles in the group diagnosed by genetic screening. However, the group of patients with clinical evidence of disease at diagnosis with the polymorphism S836S were younger than those without this allele $(20.7 \pm 8.1$ vs $33.3 \pm 13.1$ years, $P=0.03$ ). No significant differences were observed in serum calcitonin levels (540 (103.5-1800) vs 377.4 (344-410.9) pg/ml, $P=0.73$ ).

None of the RET polymorphisms were associated with the presence of PHEO, HPT, lymph node, or distant metastasis at diagnosis (Table 3). Thus, it was

Table 2 Frequency of $R E T$ polymorphisms in multiple endocrine neoplasia $2 \mathrm{~A}$ patients $(n=88)$

\begin{tabular}{llccccc}
\hline & & \multicolumn{3}{c}{ Genotype distribution } & & \\
\cline { 3 - 5 } $\begin{array}{l}\text { Sequence } \\
\text { variant }\end{array}$ & Wild-type & Heterozygous & Homozygous & $\begin{array}{c}\text { Allele } \\
\text { frequency (\%) }\end{array}$ & $\begin{array}{c}\text { Prevalence in } \\
\text { literature }\end{array}$ \\
\hline Exon 13 & L769L & 58 & 26 & 4 & 17.3 & $21.6-31$ \\
Exon 14 & S836S & 74 & 14 & 0 & 7.95 & $1-16$ \\
Exon 15 & G691S/S904S & 59 & 26 & 3 & 18.2 & $4.5-27$ \\
\hline
\end{tabular}

Fisher's exact test was used to test for Hardy-Weinberg equilibrium $(P>0.20)$.

aReferences: Elisei etal. (2004), Wiench et al. (2004), Baumgartner-Parzer et al. (2005), Wohllk et al. (2005) and Severskaya et al. (2010). 
Table 3 Clinical and oncological features of multiple endocrine neoplasia $2 \mathrm{~A}$ patients according to the presence of the polymorphic allele

\begin{tabular}{|c|c|c|c|c|c|c|c|c|c|c|}
\hline $\begin{array}{l}\text { Patients } \\
(n)\end{array}$ & $\begin{array}{l}\text { Total } \\
(88)\end{array}$ & $\begin{array}{l}\text { WT } \\
(58)\end{array}$ & $\begin{array}{l}\text { L769L } \\
(30)\end{array}$ & $P$ & $\begin{array}{l}\text { WT } \\
(74)\end{array}$ & $\begin{array}{l}\text { S836S } \\
(14)\end{array}$ & $P$ & $\begin{array}{l}\text { WT } \\
(59)\end{array}$ & $\begin{array}{l}\text { G691S/ } \\
\text { S904S (29) }\end{array}$ & $P$ \\
\hline Sex female (\%) & 56 & 60.3 & 56.7 & $0.91^{\mathrm{a}}$ & 62.2 & 42.9 & $0.29^{a}$ & 55.9 & 65.5 & $0.53^{\mathrm{a}}$ \\
\hline $\mathrm{Age}^{\mathrm{b}}$ & $27.6 \pm 15.8$ & $28.3 \pm 14.9$ & $25.1 \pm 13.1$ & 0.34 & $28.6 \pm 14.4$ & $17.0 \pm 8.2$ & 0.01 & $28.3 \pm 15.4$ & $25.0 \pm 12.1$ & 0.34 \\
\hline Pheo (\%) & 27 & 20.7 & 36.7 & $0.22^{\mathrm{a}}$ & 27.0 & 21.4 & $0.81^{\mathrm{c}}$ & 25.4 & 27.6 & $0.34^{\mathrm{a}}$ \\
\hline HPT (\%) & 17 & 15.5 & 26.7 & $0.36^{\mathrm{a}}$ & 18.9 & 21.4 & $0.89^{\mathrm{C}}$ & 22.0 & 13.8 & $0.25^{\mathrm{a}}$ \\
\hline$P N 1^{d}(\%)$ & 33 & 31.3 & 41.4 & $0.51^{\mathrm{a}}$ & 34.8 & 36.4 & $1.0^{c}$ & 40.8 & 25 & $0.25^{\mathrm{a}}$ \\
\hline$P M 1^{d}(\%)$ & 13 & 10.4 & 13.8 & $0.72^{\mathrm{c}}$ & 12.1 & 9.1 & $1.0^{\mathrm{c}}$ & 10.2 & 14.3 & $0.71^{\mathrm{c}}$ \\
\hline
\end{tabular}

Pheo, pheochromocytoma; HPT, hyperparathyroidism; PN1, lymph node metastasis; PM1, distant metastasis; WT, wild-type.

'Variables were compared using the Yates' $\chi^{2}$-test.

${ }^{\mathrm{b}}$ Age, age at diagnosis, expressed as mean \pm s.D. Variables were compared using Student's $t$-test.

'Variables were compared using the Fisher's exact test.

${ }^{\mathrm{d}}$ Data available for only 79 patients.

somewhat puzzling that patients heterozygous for the S836S allele, on average 11 years younger than wild-type subjects, presented a virtually identical percentage of lymph node and distant metastases. These results suggested to us that these events occurred earlier in individuals harboring the S836S genotype. To test this hypothesis, we have used the Kaplan-Meier model. As gene dysfunction is present since birth, we assumed that the individual age at surgery would indicate the period of exposure. Kaplan-Meier estimates of cumulative lymph node and distant metastases yielded distinct curves for patients harboring the S836S allele $(P=0.003$ and $P=0.026$ respectively, Fig. 1$)$. Kaplan-Meier analysis of cumulative metastasis for L769L and G691S/S904S genotypes yielded similar curves (data not shown).

\section{RET polymorphisms in sporadic MTC patients}

Next, we evaluated the allele frequency of the S836S polymorphism in 81 patients with sporadic MTC followed at our institution. Table 5 shows the clinical characteristics of the studied patients. The median basal serum calcitonin level at diagnosis was 682 (67.7-2650) $\mathrm{pg} / \mathrm{ml}$. The control group consisted of 80 blood donor volunteers. The mean age was $48.2 \pm 10.1$ years $(P=0.41)$, and the percentage of women were $59.3 \%(P=0.20)$. None had a recorded history of malignancy or endocrine disease. The ethnic background of both cases and controls was similar, with more than $95 \%$ of Caucasians. The frequency of the S836S allele was higher in sporadic MTC patients when compared with controls (10.5 vs $3.2 \%, P=0.01)$

Table 4 Clinical and oncological features of multiple endocrine neoplasia 2A patients diagnosed based on genetic screening or clinical grounds

\begin{tabular}{|c|c|c|c|c|c|c|c|c|c|c|}
\hline $\begin{array}{l}\text { Patients } \\
(n)\end{array}$ & $\begin{array}{l}\text { Total } \\
(33)\end{array}$ & $\begin{array}{l}\text { WT } \\
(23)\end{array}$ & $\begin{array}{l}\text { L769L } \\
(10)\end{array}$ & $\boldsymbol{P}$ & $\begin{array}{l}\text { WT } \\
(25)\end{array}$ & $\begin{array}{l}\text { S836S } \\
(8)\end{array}$ & $\boldsymbol{P}$ & $\begin{array}{l}\text { WT } \\
(25)\end{array}$ & $\begin{array}{l}\text { G691S/ } \\
\text { S904S } \\
(8)\end{array}$ & $P$ \\
\hline \multicolumn{11}{|c|}{ Genetic screening } \\
\hline Sex female (\%) & 52.9 & 52.2 & 60 & $0.72^{\mathrm{a}}$ & 60 & 37.5 & $0.41^{\mathrm{a}}$ & 52 & 62.5 & $0.70^{\circ}$ \\
\hline $\mathrm{Age}^{\mathrm{b}}$ & $14.0 \pm 7.1$ & $14.4 \pm 3.9$ & $14.4 \pm 10.4$ & 0.98 & $14.7 \pm 7.3$ & $13.5 \pm 6.5$ & 0.71 & $14.4 \pm 7.3$ & $14.3 \pm 6.7$ & 0.98 \\
\hline$n$ & $(55)$ & (35) & $(20)$ & $P$ & $(49)$ & (6) & $\boldsymbol{P}$ & (34) & (21) & $\boldsymbol{P}$ \\
\hline \multicolumn{11}{|l|}{ Clinical disease } \\
\hline Sex female (\%) & 58.2 & 65.7 & 55 & $0.62^{c}$ & 63.3 & 50 & $0.66^{a}$ & 58.8 & 66.7 & $0.77^{\circ}$ \\
\hline $\mathrm{Age}^{\mathrm{b}}$ & $32.1 \pm 13.1$ & $33.6 \pm 14.0$ & $29.8 \pm 11.4$ & 0.31 & $33.3 \pm 13.1$ & $20.7 \pm 8.1$ & 0.03 & $34.5 \pm 13.8$ & $28.6 \pm 11.3$ & 0.15 \\
\hline Pheo (\%) & $40.3^{-}$ & $34.3^{-}$ & 55 & $0.23^{c}$ & 40.8 & 50 & $0.87^{a}$ & 44.1 & 38.1 & $0.36^{\mathrm{c}}$ \\
\hline HPT (\%) & 23.9 & 22.9 & 40 & $0.29^{c}$ & 26.5 & 50 & $0.47^{\mathrm{a}}$ & 35.3 & 19 & $0.22^{\circ}$ \\
\hline$P N 1^{d}(\%)$ & 53 & 42.9 & 60 & $0.34^{c}$ & 46.9 & 66.7 & $0.42^{\mathrm{a}}$ & 58.8 & 33.3 & $0.11^{\mathrm{c}}$ \\
\hline$P M 1^{d}(\%)$ & 19.4 & 14.3 & 20 & $0.71^{a}$ & 16.3 & 16.7 & $1.00^{\mathrm{a}}$ & 14.7 & 19 & $0.72^{\mathrm{a}}$ \\
\hline
\end{tabular}

Pheo, pheochromocytoma; HPT, hyperparathyroidism; PN1, lymph node metastasis; PM1, distant metastasis; WT, wild-type.

aVariables were compared using the Fisher's exact test.

${ }^{\mathrm{b}} \mathrm{Age}$, age at diagnosis, expressed as mean \pm S.D. Variables were compared using Student's $t$-test.

'Variables were compared using the Yates' $\chi^{2}$-test.

dData available for only 46 patients. 

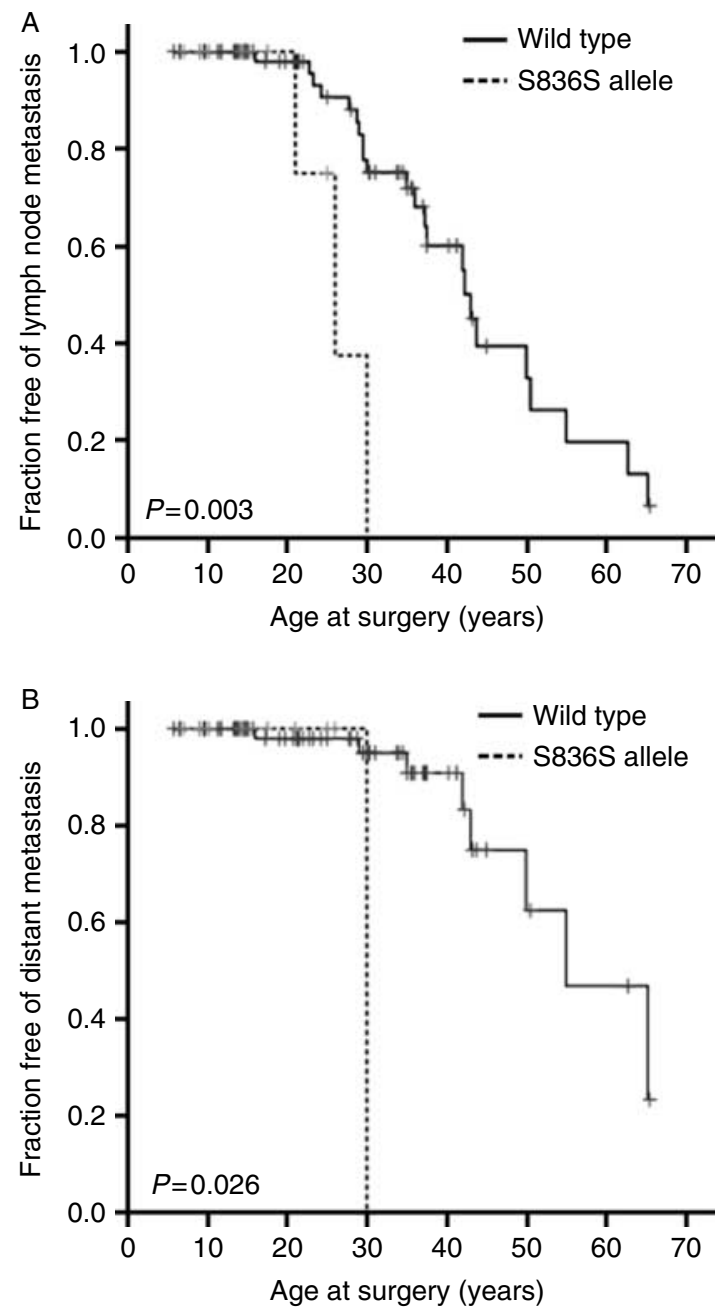

Figure 1 Kaplan-Meier estimates of the proportion of hereditary MTC patients $(n=79)$ with lymph node $(\mathrm{A})$ or distant metastasis $(B)$ at diagnosis. *The log rank test was used to compare curves.

but similar to that observed in the hereditary group $(7.95 \%, P=0.43)$. Genotypes were in Hardy-Weinberg equilibrium $(P>0.20)$.

Of the 81 MTC patients, 64 patients $(79 \%)$ were homozygous for the wild-type allele (CC), and 17 patients $(21 \%)$ were heterozygous for S836S polymorphic allele. There was no homozygosis for the S836S polymorphic allele. Individuals harboring the S836S variant were significantly younger at diagnosis than those without this allele $(38.6 \pm 13.3$ vs $48.5 \pm 16.7$ years, $P=0.02$ ), had higher serum calcitonin level (4193 (1600-13 737) vs 539 (28.2-1440) $\mathrm{pg} / \mathrm{ml}, P=0.005$ ), and presented a higher percentage of lymph node and distant metastases at diagnosis $(81.3$ vs 45.9 and 43.8 vs $17.7 \%, P=0.02$ and $P=0.04$ respectively). Accordingly, Kaplan-Meier estimates of cumulative lymph node and distant metastases yielded distinct curves for patients with or without the S836S allele ( $P=0.002$ and $P=0.001$ respectively, Fig. 2$)$, further demonstrating that metastatic disease occurred earlier in those individuals harboring the S836S variant.

\section{Somatic M918T mutation analysis}

The following step was to look for somatic RET M918T mutation in the sporadic MTC group, since it has been shown that the presence of this missense somatic RET mutation correlates with the presence of lymph node metastases at diagnosis (Elisei et al. 2008) and could be a confounding factor in our analysis.

Forty paraffin-embedded MTC samples were available. Of them, we were unable to extract DNA from nine samples even after several repeated attempts. Of the 31 DNA samples available for analysis, 25 samples (80.6\%) were found to have somatic M918T. Although we observed a higher frequency of the S836S allele in this subgroup of patients $(11 / 31,35.5 \%)$, there was no significant association between somatic M918T mutation and S836S polymorphic allele (10/25 (40\%) vs $1 / 6(16.7 \%)$ respectively, $P=0.38)$.

\section{S836S polymorphism is an independent risk factor for metastatic disease in MTC patients}

Because previous studies demonstrated that specific nucleotide and amino acid exchanges at codon 634 might have a direct impact on tumor aggressiveness in MEN 2A (Punales et al. 2003, Milos et al. 2008), we speculated whether co-segregation with a specific $R E T$ mutation could interfere with our results.

According to the germline mutation, the 14 patients harboring the S836S polymorphic allele were distributed as following: 10 out of 62 individuals with C634Y mutation (3 kindred) and 4 out of 7 individuals with $\mathrm{C} 634 \mathrm{~W}$ mutation (1 kindred). We found that in all

Table 5 Clinical and oncological features of sporadic MTC patients according to the presence of the S836S polymorphic allele

\begin{tabular}{lllll}
\hline Patients $(n)$ & Total (81) & WT (64) & S836S (17) & $\boldsymbol{P}$ \\
\hline Sex female (\%) & 59.3 & 59.4 & 58.8 & $1.0^{\mathrm{a}}$ \\
Age $^{\mathrm{b}}$ & $27.6 \pm 15.8$ & $48.5 \pm 16.7$ & $38.6 \pm 13.3$ & 0.02 \\
$\mathrm{PN1}^{\mathrm{c}}(\%)$ & 50.6 & 45.9 & 81.3 & $0.02^{\mathrm{a}}$ \\
$\mathrm{PM}^{\mathrm{c}}(\%)$ & 22.2 & 17.7 & 43.8 & $0.04^{\mathrm{d}}$ \\
\hline
\end{tabular}

PN1, lymph node metastasis; PM1, distant metastasis; WT, wild-type.

'Variables were compared using the Yates' $\chi^{2}$-test.

${ }^{b}$ Age, age at diagnosis, expressed as mean \pm s.D. Variables were compared using Student's $t$-test.

'Data available for 74 patients.

dVariables were compared using the Fisher's exact test. 
cases, the S836S variant did not co-segregate with the germline mutation and was inherited from the unaffected parent.

To further evaluate whether the effect of the S836S variant allele was associated with a specific germline mutation, we used the Cox proportional hazard survival analysis with the presence of metastasis at diagnosis as the outcome and the survival time as the age at diagnosis of MTC. To increase the statistical power of the analysis, all patients with MTC diagnosis (hereditary and sporadic groups) were included $(n=153)$. The results are shown in Table 6. The presence of the neutral RET S836S variant was an independent risk factor for early local or distant metastatic disease in MTC. As expected, all germline mutations except C618R were significantly associated with increased risk for early metastatic disease (Table 6). The lack of significance in the C618R association was probably due to the small number of subjects harboring this mutation (nine individuals).
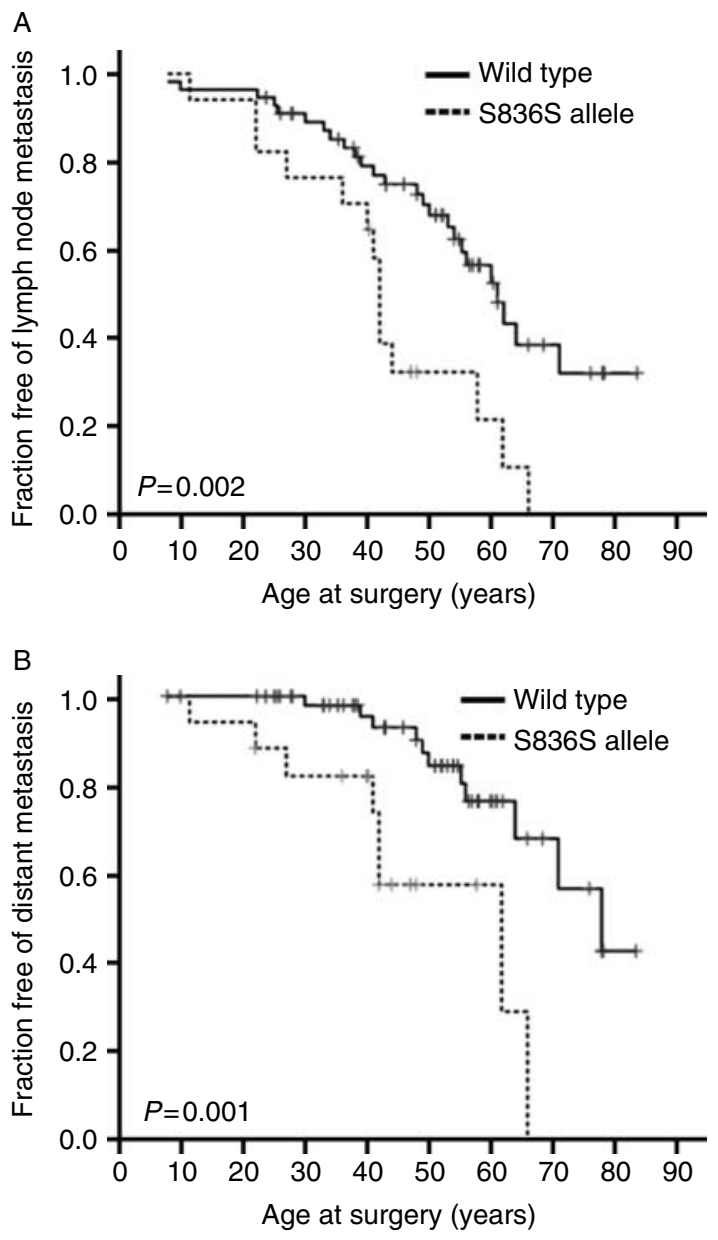

Figure 2 Kaplan-Meier estimates of the proportion of sporadic MTC patients $(n=74)$ with lymph node (A) or distant metastasis (B) at diagnosis. *The log rank test was used to compare curves.
Table 6 Analysis of survival Cox regression $(n=153)$

\begin{tabular}{lcccc}
\hline Variables & $\boldsymbol{b}$ & $\boldsymbol{P}$ & HR & Cl \\
\hline S836S & 1.04 & 0.001 & 2.82 & $(1.51-5.26)$ \\
C634W & 1.79 & 0.007 & 6.03 & $(1.63-22.4)$ \\
C634Y & 0.76 & 0.014 & 2.14 & $(1.17-3.93)$ \\
C634R & 1.90 & $<0.005$ & 6.69 & $(2.45-18.3)$ \\
C618R & 0.67 & 0.27 & 1.96 & $(0.59-6.51)$ \\
\hline
\end{tabular}

$b$, regression coefficient; $\mathrm{HR}$, hazard ratio; $\mathrm{Cl}$, confidence interval.

\section{Discussion}

In the present study, we have demonstrated that the neutral RET polymorphism S836S is associated with early onset and increased risk for metastatic disease at a younger age in individuals with hereditary or sporadic MTC. Patients harboring the variant allele were younger at diagnosis and presented with early local and distant metastasis, as assessed by the Kaplan-Meier model. Moreover, additional analysis using multivariate Cox regression identified this polymorphism as an independent risk factor for lymph node or distant metastasis.

Several SNPs of the RET proto-oncogene have been described in the general population as well as in patients with familial and sporadic MTC (Gimm et al. 1999, Ruiz et al. 2001, Elisei et al. 2004, Severskaya et al. 2010, Tamanaha et al. 2009). Here, we have studied the frequency of the exonic RET polymorphisms $L 769 L$, S836S, and G691S/S904S in 17 MEN 2A families. We did not detect significant differences related to disease phenotype or tumor stage. However, we observed that patients harboring the S836S variant allele were about 11 years younger than those with the wild-type genotype (Table 3). To rule out selection bias on the age of diagnosis due to genetic screening, patients were analyzed separately based on the presence or the absence of clinical disease at diagnosis. Of particular note, no differences in age at diagnosis in the group were diagnosed by genetic screening, but the positive association between the polymorphism S836S and earlier age at diagnosis remained in the group with clinical evidence of disease at diagnosis (Table 4). Surprisingly, despite that difference in age at diagnosis, patients with or without the polymorphic allele displayed a virtually identical percentage of lymph node and distant metastases (Table 3). Thus, we speculate that these individuals will develop metastasis at a younger age. Accordingly, Kaplan-Meier estimates of cumulative metastasis yielded distinct curves, indicating that these events occurred earlier in individuals with the S836S polymorphic allele (Fig. 1). 
The presence of the S836S variant allele was also associated with younger age and a higher percentage of local and distant metastases at diagnosis in a sample of sporadic MTC patients (Table 5 and Fig. 2). A possible role of this variant in the pathogenesis of sporadic MTC has been speculated by several studies. Similar to the findings of this study, the S836S variant allele was over-represented in sporadic MTC patients from Germany, Spain, and the United States (Gimm et al. 1999, Ruiz et al. 2001), but no differences were observed between controls and French, Polish, British, Chilean, Portuguese, and Austrian patients (Wiench et al. 2001, Berard et al. 2004, Baumgartner-Parzer et al. 2005, Cebrian et al. 2005, Costa et al. 2005, Wohllk et al. 2005). The reasons for these conflicting results are still unclear. Yet, a significantly higher frequency of the S836S variant in patients with M918T somatic mutation in sporadic MTC has been reported (Gimm et al. 1999). Of interest, a kindred where the carriers of S836S developed MTC (Gimm et al. 1999) and a case of C-cell hyperplasia and primary HPT in an individual harboring the S836S polymorphism in the absence of germline RET mutations have also been reported (Brauckhoff et al. 2002).

In contrast to the large number of studies focused on the role of S836S variant in sporadic MTC, we have found only a few focused on hereditary MTC. Tamanaha et al. (2009) demonstrated that the S836S variant was over-represented in G533C carriers and non-carrier family members compared to the control population. However, they were unable to show an association between this variant and earlier age at onset in the large kindred cohort studied (Tamanaha et al. 2009). A modulating effect of the combination of polymorphic $L 769 L$ with wild-type S836S on the clinical outcome of hereditary MTC has also been described (Severskaya et al. 2010). The segregation of RET V804L germline mutation and the S836S variant was reported in a Hungarian FMTC kindred comprising 80 individuals of four generations, but the co-existence of the V804L mutation and S836S polymorphism did not seem to aggravate the relatively low-risk disease phenotype (Patocs et al. 2003).

The exact mechanism by which these polymorphisms modulate MTC pathogenesis or disease presentation is still not known and is open to speculation. Even though these allelic variants do not seem to confer any transforming activity on the tyrosine kinase domain of the RET protein, cumulative studies suggest that they might modify disease susceptibility and clinical phenotype in patients with sporadic or hereditary MTC. Because it has been reported that polymorphic sequence variants can lead to the production of different amounts of mRNA, a theoretical role for the polymorphisms in the pathogenesis of MTC is that the allelic variant might influence RET mRNA expression. However, Elisei et al. (2004) did not find any significant difference in the levels of RET mRNA when comparing sporadic MTC patients with or without G691S/S904S, L769L, or S836S polymorphism. The S836S polymorphism failed to affect DNA-protein binding, transcript stability, or RNA splicing and editing (Griseri et al. 2000), but it is possible that this genetic variant may create an unstable sequence upstream or downstream at germline or somatic RET mutations instead of directly participating in the tumorigenic process (Gimm et al. 1999). Such a mechanism has been observed in the $A P C$ gene in a fraction (28\%) of Ashkenazim with familial colorectal cancer where additional somatic mutations were more often found on that allele carrying a seemingly innocuous germline missense mutation predicted to result in a conservative amino acid change (Laken et al. 1997).

A novel observation in this study was the association between the S836S polymorphic allele and early metastatic disease observed in both hereditary and sporadic MTC, suggesting that this variant might interfere in tumor progression. The S836S allele did not co-segregate with the germline mutation and was aleatorily distributed among individuals with 634 mutations, the most prevalent mutation in our series. Accordingly, statistical analysis performed using the Cox proportional hazard survival model identified the S836S variant as an independent risk factor for metastatic disease in MTC (hazard ratio 2.82, Table 6). A possible confounding effect of somatic M918T mutation in the sporadic group was also addressed. The M918T mutation was present in about $80 \%$ of the samples analyzed, a frequency similar to that reported in some centers (Zedenius et al. 1995, Marsh et al. 1996, Moura et al. 2009), but that could be influenced by selection since most of the available samples were from patients followed at our institution due to advanced disease. Although we observed a much higher frequency of the S836S variant (35\%) in this subgroup of patients, there was no significant association between somatic M918T mutation and the S836S polymorphic allele $(P=0.383)$.

As previously discussed, the mechanistic explanation is speculative. An additional hypothesis is that an unknown functional variant, which may be in linkage disequilibrium with the haplotype containing the S836S variant, could possibly control the activity of the RET oncogene. An MTC-specific risk haplotype that includes the S836S and IVS1-126G $>\mathrm{T}$ RET 
polymorphisms was previously described (Borrego et al. 2003). The IVS1-126G $>\mathrm{T}$ variant has also been associated with the development of sporadic MTC (Fernandez et al. 2004) and, interestingly, with earlier age at disease onset in a large Brazilian kindred cohort harboring the G533C RET mutation (Tamanaha et al. 2009). Of note, an in silico analysis revealed that this genetic variant creates a new binding site for NFAT transcription factor (nuclear factor of activated T-cells (Borrego et al. 2003). The NFAT family of proteins has been found to be involved in cell cycle regulation, cell differentiation, cell survival, angiogenesis, tumor cell invasion, and metastasis (Lu \& Huan 2007).

The RET variants $L 769 L$ and G691S/S904S have also been studied as modifiers in disease presentation in both hereditary and sporadic MTC patients (Berard et al. 2004, Fernandez et al. 2006a,b, Guerrero et al. 2006). The polymorphic G691S/S904S variant of RET has been implicated as a modifier factor on the age at which MEN 2A begins (Gil et al. 2002, Robledo et al. 2003), whereas the RETL769L polymorphism has been previously implicated as having an effect in the early development of hereditary MTC in a family with a mutation in exon 14 (Magalhaes et al. 2004). However, we did not observe an association between the presence of these variants and clinical presentation of the disease in hereditary MTC. This highlights the importance of replication in different populations and might indicate differences due to genetic background or geographic areas.

Some factors unrelated to the RET polymorphisms could have interfered with the findings of the present study. Firstly, our results could represent a type 1 error. However, the S836S was significantly associated to younger age at diagnosis and early metastatic disease in patients with clinical disease in hereditary and sporadic MTC groups. These results argue against an association by chance. Secondly, due to the relatively small percentage of cases (38\%) analyzed, we cannot formally rule out a confounding effect from the somatic M918T mutation in the sporadic group. Finally, this study has insufficient statistical power to exclude the possibility of an association between the $L 769 \mathrm{~L}$ or G691S/S904S variants and clinical presentation of the disease in hereditary MTC (type 2 error).

In conclusion, our data indicate that the RET variant S836S is associated with increased risk for metastatic disease at a younger age in individuals with MEN 2A or sporadic MTC. If confirmed in other sample populations, these findings might have significant implications on the management of MTC, particularly on defining the ideal timing for prophylactic intervention on gene carriers.

\section{Declaration of interest}

The authors declare that there is no conflict of interest that could be perceived as prejudicing the impartiality of the research reported.

\section{Funding}

This work was supported by Conselho Nacional de Desenvolvimento Científico e Tecnológico (CNPq), Fundação de Amparo Pesquisa do Estado do Rio Grande do Sul (FAPERGS), and Fundo de Incentivo à Pesquisa do Hospital de Clínicas de Porto Alegre (FIPE), Brazil.

\section{References}

Baumgartner-Parzer SM, Lang R, Wagner L, Heinze G, Niederle B, Kaserer K, Waldhausl W \& Vierhapper H 2005 Polymorphisms in exon 13 and intron 14 of the RET protooncogene: genetic modifiers of medullary thyroid carcinoma? Journal of Clinical Endocrinology and Metabolism 90 6232-6236. (doi:10.1210/jc.2005-1278)

Berard I, Kraimps JL, Savagner F, Murat A, Renaudin K, Nicolli-Sire P, Bertrand G, Moisan JP \& Bezieau S 2004 Germline-sequence variants S836S and L769L in the RE arranged during transfection (RET) proto-oncogene are not associated with predisposition to sporadic medullary carcinoma in the French population. Clinical Genetics $\mathbf{6 5}$ 150-152. (doi:10.1111/j.0009-9163.2004.00172.x)

Borrego S, Wright FA, Fernandez RM, Williams N, LopezAlonso M, Davuluri R, Antinolo G \& Eng C 2003 A founding locus within the RET proto-oncogene may account for a large proportion of apparently sporadic Hirschsprung disease and a subset of cases of sporadic medullary thyroid carcinoma. American Journal of Human Genetics 72 88-100. (doi:10.1086/345466)

Brauckhoff M, Gimm O, Bilkenroth U, Hinze R \& Dralle H 2002 Primary hyperparathyroidism, C-cell hyperplasia and papillary thyroid carcinoma in a patient with RET germline polymorphism S836S. Langenbeck's Archives of Surgery 387 201-203. (doi:10.1007/s00423-002-0321-y)

Cebrian A, Lesueur F, Martin S, Leyland J, Ahmed S, Luccarini C, Smith PL, Luben R, Whittaker J, Pharoah PD et al. 2005 Polymorphisms in the initiators of RET (rearranged during transfection) signaling pathway and susceptibility to sporadic medullary thyroid carcinoma. Journal of Clinical Endocrinology and Metabolism 90 6268-6274. (doi:10.1210/jc.2004-2449)

Costa P, Domingues R, Sobrinho LG \& Bugalho MJ 2005 RET polymorphisms and sporadic medullary thyroid carcinoma in a Portuguese population. Endocrine 27 239-243. (doi:10.1385/ENDO:27:3:239)

Elisei R, Cosci B, Romei C, Bottici V, Sculli M, Lari R, Barale R, Pacini F \& Pinchera A 2004 RET exon 11 (G691S) polymorphism is significantly more frequent in sporadic medullary thyroid carcinoma than in the general 
population. Journal of Clinical Endocrinology and Metabolism 89 3579-3584. (doi:10.1210/jc.2003031898)

Elisei R, Cosci B, Romei C, Bottici V, Renzini G, Molinaro E, Agate L, Vivaldi A, Faviana P, Basolo F et al. 2008 Prognostic significance of somatic RET oncogene mutations in sporadic medullary thyroid cancer: a 10-year follow-up study. Journal of Clinical Endocrinology and Metabolism 93 682-687. (doi:10.1210/jc.2007-1714)

Eng C, Clayton D, Schuffenecker I, Lenoir G, Cote G, Gagel RF, van Amstel HK, Lips CJ, Nishisho I, Takai SI et al. 1996 The relationship between specific RET proto-oncogene mutations and disease phenotype in multiple endocrine neoplasia type 2 . International RET mutation consortium analysis. Journal of the American Medical Association 276 1575-1579. (doi:10.1001/jama. 276.19.1575)

Fernandez RM, Robledo M, Antinolo G, Pecina A, Ruiz-Llorente S, Eng C \& Borrego S 2004 The RET IVS1-126G $>$ T variant is strongly associated with the development of sporadic medullary thyroid cancer. Thyroid 14 329-331. (doi:10.1089/1050725043230 31022)

Fernandez RM, Navarro E, Antinolo G, Ruiz-Ferrer M \& Borrego S 2006a Evaluation of the role of RET polymorphisms/haplotypes as modifier loci for MEN 2, and analysis of the correlation with the type of RET mutation in a series of Spanish patients. International Journal of Molecular Medicine 17 575-581.

Fernandez RM, Pecina A, Antinolo G, Navarro E \& Borrego S $2006 b$ Analysis of RET polymorphisms and haplotypes in the context of sporadic medullary thyroid carcinoma. Thyroid 16 411-417. (doi:10.1089/thy.2006.16.411)

Gil L, Azanedo M, Pollan M, Cristobal E, Arribas B, GarciaAlbert L, Garcia-Saiz A, Maestro ML, Torres A, Menarguez J et al. 2002 Genetic analysis of RET, GFR alpha 1 and GDNF genes in Spanish families with multiple endocrine neoplasia type 2A. International Journal of Cancer 99 299-304. (doi:10.1002/ijc.10298)

Gimm O, Neuberg DS, Marsh DJ, Dahia PL, Hoang-Vu C, Raue F, Hinze R, Dralle H \& Eng C 1999 Overrepresentation of a germline RET sequence variant in patients with sporadic medullary thyroid carcinoma and somatic RET codon 918 mutation. Oncogene 18 1369-1373. (doi:10.1038/sj.onc.1202418)

Griseri P, Sancandi M, Patrone G, Bocciardi R, Hofstra R, Ravazzolo R, Devoto M, Romeo G \& Ceccherini I 2000 A single-nucleotide polymorphic variant of the RET protooncogene is underrepresented in sporadic Hirschsprung disease. European Journal of Human Genetics 8 721-724. (doi:10.1038/sj.ejhg.5200521)

Guerrero IM, Pessoa CH, Olmedo DB, Pontes ER, Matos LC, Tilli TM, Barcinski MA \& Gimba ER 2006 Analysis of inherited genetic variants in ret proto-oncogene of Brazilian patients with apparently sporadic medullary thyroid carcinoma. Thyroid 16 9-15. (doi:10.1089/thy. 2006.16.9)
Kouvaraki MA, Shapiro SE, Perrier ND, Cote GJ, Gagel RF, Hoff AO, Sherman SI, Lee JE \& Evans DB 2005 RET proto-oncogene: a review and update of genotypephenotype correlations in hereditary medullary thyroid cancer and associated endocrine tumors. Thyroid $\mathbf{1 5}$ 531-544. (doi:10.1089/thy.2005.15.531)

Laken SJ, Petersen GM, Gruber SB, Oddoux C, Ostrer H, Giardiello FM, Hamilton SR, Hampel H, Markowitz A, Klimstra D et al. 1997 Familial colorectal cancer in Ashkenazim due to a hypermutable tract in APC. Nature Genetics 17 79-83. (doi:10.1038/ng0997-79)

Lesueur F, Cebrian A, Robledo M, Niccoli-Sire P, Svensson KA, Pinson S, Leyland J, Whittaker J, Pharoah PD \& Ponder BA 2006 Polymorphisms in RET and its coreceptors and ligands as genetic modifiers of multiple endocrine neoplasia type 2A. Cancer Research 66 1177-1180. (doi:10.1158/0008-5472.CAN-05-2995)

Lu H \& Huan C 2007 Transcription factor NFAT, its role in cancer development, and as a potential target for chemoprevention. Current Cancer Drug Targets 7 343-353. (doi:10.2174/156800907780809750)

Machens A, Gimm O, Hinze R, Hoppner W, Boehm BO \& Dralle H 2001 Genotype-phenotype correlations in hereditary medullary thyroid carcinoma: oncological features and biochemical properties. Journal of Clinical Endocrinology and Metabolism 86 1104-1109. (doi:10. 1210/jc.86.3.1104)

Machens A, Niccoli-Sire P, Hoegel J, Frank-Raue K, van Vroonhoven TJ, Roeher HD, Wahl RA, Lamesch P, Raue F, Conte-Devolx B et al. 2003 Early malignant progression of hereditary medullary thyroid cancer. New England Journal of Medicine 349 1517-1525. (doi:10.1056/NEJMoa012915)

Magalhaes PK, de Castro M, Elias LL, Soares EG \& Maciel LM 2004 Polymorphisms in the RET proto-oncogene and the phenotypic presentation of familial medullary thyroid carcinoma. Thyroid 14 848-852. (doi:10.1089/thy.2004. 14.848)

Marsh DJ, Learoyd DL, Andrew SD, Krishnan L, Pojer R, Richardson AL, Delbridge L, Eng C \& Robinson BG 1996 Somatic mutations in the RET proto-oncogene in sporadic medullary thyroid carcinoma. Clinical Endocrinology 44 249-257. (doi:10.1046/j.1365-2265.1996.681503.x)

Milos IN, Frank-Raue K, Wohllk N, Maia AL, Pusiol E, Patocs A, Robledo M, Biarnes J, Barontini M, Links TP et al. 2008 Age-related neoplastic risk profiles and penetrance estimations in multiple endocrine neoplasia type 2A caused by germ line RET Cys634Trp (TGC > TGG) mutation. Endocrine-Related Cancer 15 1035-1041. (doi:10.1677/ERC-08-0105)

Moura MM, Cavaco BM, Pinto AE, Domingues R, Santos JR, Cid MO, Bugalho MJ \& Leite V 2009 Correlation of RET somatic mutations with clinicopathological features in sporadic medullary thyroid carcinomas. British Journal of Cancer 100 1777-1783. (doi:10.1038/sj.bjc. 6605056) 
O’Sullivan B \& Shah J 2003 New TNM staging criteria for head and neck tumors. Seminars in Surgical Oncology 21 30-42. (doi:10.1002/ssu.10019)

Patocs A, Valkusz Z, Igaz P, Balogh K, Toth M, Varga I \& Racz K 2003 Segregation of the V804L mutation and S836S polymorphism of exon 14 of the RET gene in an extended kindred with familial medullary thyroid cancer. Clinical Genetics 63 219-223. (doi:10.1034/j.1399-0004. 2003.00044.x)

Ponder BA 1999 The phenotypes associated with ret mutations in the multiple endocrine neoplasia type 2 syndrome. Cancer Research 59 1736s-1741s (discussion 1742s).

Punales MK, Graf H, Gross JL \& Maia AL 2003 RET codon 634 mutations in multiple endocrine neoplasia type 2 : variable clinical features and clinical outcome. Journal of Clinical Endocrinology and Metabolism 88 2644-2649. (doi:10.1210/jc.2002-021422)

Punales MK, da Rocha AP, Meotti C, Gross JL \& Maia AL 2008 Clinical and oncological features of children and young adults with multiple endocrine neoplasia type $2 \mathrm{~A}$. Thyroid 18 1261-1268. (doi:10.1089/thy.2007.0414)

Robledo M, Gil L, Pollan M, Cebrian A, Ruiz S, Azanedo M, Benitez J, Menarguez J \& Rojas JM 2003 Polymorphisms G691S/S904S of RET as genetic modifiers of MEN 2A. Cancer Research 63 1814-1817.

Rocha AP, Magalhaes PK, Maia AL \& Maciel LM 2007 Genetic polymorphisms: implications in the pathogenesis of medullary thyroid carcinoma. Arquivos Brasileiros de Endocrinologia e Metabologia 51 723-730. (doi:10.1590/ S0004-27302007000500009)

Ruiz A, Antinolo G, Fernandez RM, Eng C, Marcos I \& Borrego S 2001 Germline sequence variant S836S in the RET proto-oncogene is associated with low level predisposition to sporadic medullary thyroid carcinoma in the Spanish population. Clinical Endocrinology 55 399-402. (doi:10.1046/j.1365-2265.2001.01328.x)
Severskaya NV, Saenko VA, Ilyin AA, Chebotareva IV, Rumyantsev PO, Isaev PA, Medvedev VS \& Yamashita S 2010 Germline polymorphisms of RET and GFRA1 genes in patients with medullary thyroid carcinoma. Molecular Biology 40 375-384. (doi:10.1134/S0026893306030046)

Tamanaha R, Camacho CP, Pereira AC, da Silva AM, Maciel RM \& Cerutti JM 2009 Evaluation of RET polymorphisms in a six-generation family with G533C RET mutation: specific RET variants may modulate age at onset and clinical presentation. Clinical Endocrinology 71 56-64. (doi:10.1111/j.1365-2265.2008.03491.x)

Wiench M, Wygoda Z, Gubala E, Wloch J, Lisowska K, Krassowski J, Scieglinska D, Fiszer-Kierzkowska A, Lange D, Kula D et al. 2001 Estimation of risk of inherited medullary thyroid carcinoma in apparent sporadic patients. Journal of Clinical Oncology 19 1374-1380.

Wiench M, Wloch J, Wygoda Z, Gubala E, Oczko M, Pawlaczek A, Kula D, Lange D \& Jarzab B 2004 RET polymorphisms in codons 769 and 836 are not associated with predisposition to medullary thyroid carcinoma. Cancer Detection and Prevention 28 231-236. (doi:10. 1016/j.cdp.2004.04.002)

Wohllk N, Soto E, Bravo M \& Becker P 2005 Polimorfismos G691S, L769L y S836S del proto-oncogene RET no se associan a mayor riesgo de cancer medular tiroideo esporádico en pacientes chilenos. Revista Mèdica de Chile 133 397-402. (doi:10.4067/S0034-98872005000 400001)

Zedenius J, Larsson C, Bergholm U, Bovee J, Svensson A, Hallengren B, Grimelius L, Backdahl M, Weber G \& Wallin G 1995 Mutations of codon 918 in the RET protooncogene correlate to poor prognosis in sporadic medullary thyroid carcinomas. Journal of Clinical Endocrinology and Metabolism 80 3088-3090. (doi:10. 1210/jc.80.10.3088) 